American Journal of Infectious Diseases 3 (4): 255-266, 2007

ISSN 1553-6203

(C) 2007 Science Publications

\title{
Effects of Bacillus subtilis 'PB6' (ATCC - PTA 6737) on Clostridium difficile Associated Diarrhea (CDAD) and Inflammatory Bowel Disease (IBD) in Animal Models
}

\author{
${ }^{1}$ Eric Peys, ${ }^{2}$ Jerry Varghese, ${ }^{2}$ Ponusami Suresh, ${ }^{1}$ Jan Vandenkerckhove, ${ }^{1}$ Johan Van hemel, \\ ${ }^{2}$ Ramchand Nanappan Chaniyilparampu and ${ }^{1}$ Benedikt Sas \\ Kemin Pharma, Atealaan 4H, 2200 Herentals, Belgium ${ }^{1}$ and Kemin Nutritional Technologies, K3, 11th \\ Cross Street, SIPCOT Industrial Complex, Gummidipundi 601201, Tamilnadu, India
}

\begin{abstract}
The administration of probiotic bacteria is emerging as a potential means of preventing the onset or recurrence of Clostridium difficile associated diarrhea (CDAD) and of attenuating inflammatory activity and preventing relapses in inflammatory bowel disease (IBD). We evaluated the efficacy of Bacillus subtilis 'PB6' (ATCC - PTA 6737) in a hamster model of antibiotic-induced CDAD and in a rat model of IBD. CDAD was induced in male Golden Syrian hamsters using $C$. difficile and clindamycin. These hamsters received either nothing or, by gavage, vancomycin (5 days) or PB6 (low, middle and high dose, 6 days). Diarrhea, body weight loss and mortality were observed in all groups in which CDAD was induced. Intensity of diarrhea and body weight loss was least in the groups treated with vancomycin or with the highest dose of PB6. At the end of the treatment period, vancomycin and the highest dose of PB6 were equally efficient in preventing mortality in this hamster model of CDAD. No adverse effects of PB6 treatment were observed in healthy animals. In male Wistar rats, colitis was induced using a single intrarectal administration of 2,4,6trinitrobenzenesulfonic acid (TNBS). Treatments consisted of PB6 (low, middle and high dose), Saccharomyces boulardii, mesalazine, infliximab, or no treatment. A possible benefit of the prophylactic use of PB6 was also tested. At the end of the treatment period significant differences in body weight gain, in colon inflammatory edema and in gross morphology of the colon intestinal lining were observed between groups. The groups treated with high dose PB6 could not be distincted from the colitis-free negative control group nor from the group treated with mesalazine. The data presented are suggestive of possible therapeutic effectiveness of PB6 in CDAD and IBD in humans.
\end{abstract}

Key words: Bacillus subtilis, Clostridium difficile associated diarrhea (CDAD), inflammatory bowel disease (IBD), animal model

\section{INTRODUCTION}

In 1965 the term "probiotics" was used for the first time to indicate "substances produced by microorganisms which promote the growth of other microorganisms" ${ }^{\text {"[1] }}$. Since then, the definition of probiotics has been modified several times. A widely used definition, developed by the FAO/WHO, calls probiotics "defined, live microorganisms administered in adequate amounts which confer to a beneficial physiological effect in the host".

Research and trials have shown that probiotics are or may become an effective therapy for gastrointestinal infections, inflammatory bowel disease (IBD) and irritable bowel syndrome (IBS) ${ }^{[2]}$.

PB6 (ATCC-PTA 6737, Anaeban ${ }^{\mathrm{TM}}$, Kemin Pharma) is a unique and natural Bacillus subtilis strain, that has shown in vitro to produce antimicrobial substances broadly active against various strains of Campylobacter sp. and Clostridium sp. and more specifically against $C$. difficile (27 November 2002, US Patent Office).

Clostridium difficile was first isolated by Hall and O' Toole in $1935^{[3]}$ and originally named Bacillus difficilis. In 1938 this species was reclassified as $C$. difficile (type strain ATCC 9689). Only in the late 1970 's, it was associated with antibiotic-related diarrhea. $C$. difficile is a spore-forming gram-positive anaerobic bacillus. Toxigenic strains can produce at least two exotoxins, virulence factors responsible for $C$. difficile disease: toxin $\mathrm{A}$, primarily an enterotoxin and toxin B, a cytotoxin. Colonization occurs via the fecaloral route. Ingested spores of $C$. difficile survive the gastric acid barrier and germinate in the colon ${ }^{[4,5]}$. 
C. difficile is the primary cause of hospital-acquired infectious diarrhea and colitis when patients receive antibiotics, chemotherapeutics or other drugs that alter their normal intestinal flora. Antibiotics most frequently associated with the infection are clindamycin, ampicillin, amoxicillin and cephalosporins, however all antibiotics might predispose a patient to $C$. difficile infection $^{[6,7]}$. The clinical presentation varies from asymptomatic colonization to mild diarrhea to severe debilitating disease, with high fever, severe abdominal pain, pseudomembranous colitis, toxic megacolon, colonic perforation, or even death ${ }^{[8-10]}$. Clostridium difficile associated diarrhea (CDAD) is currently treated with metronidazole or vancomycin. Although both are highly effective, there is a strong medical need for alternative and better therapeutic options to combat CDAD. The main factors for this are undesirable side effects associated with their use, reported metronidazole-resistant $C$. difficile isolates, spread of vancomycin-resistant enterococci in vancomycintreated patients and the fact that approximately $15-35 \%$ of the patients experience relapse after initial therapy ${ }^{[11-}$ 13].

The minimal inhibitory concentration of a PB6 fermentation broth diethyl ether crude extract against $C$. difficile ATCC 9689 was between 5 and 10 ppm. The antimicrobial actives identified in the PB6 fermentation product were surfactins. These are amphiphatic cyclic lipoheptapeptides belonging to the nonribosomal peptide family of natural products that also includes other important therapeutic agents, such as vancomycin and daptomycin. Surfactins have exceptional emulsifying but also antibacterial, antiviral and antitumoral properties ${ }^{[14-18]}$. As inhibitors of cytosolic phospholipase $\mathrm{A}_{2}\left(\mathrm{PLA}_{2}\right)$, surfactins are also putative anti-inflammatory agents ${ }^{[19]}$. Oral bacteriotherapy with PB6 therefore could deliver a potential therapeutic benefit in pathologies involving inflammation of the intestinal wall.

Based on the association of intestinal inflammation with luminal bacteria, considerable research is focused on modifying the intestinal flora with probiotic bacteria to antagonise pathogenic bacteria and/or to attenuate inflammatory activity, induce remission and prevent relapses in ulcerative colitis, Crohn's disease and pouchitis $^{[20-24]}$.

Ulcerative colitis, Crohn's disease and pouchitis are chronic inflammatory disorders of the bowel categorised as inflammatory bowel diseases. In genetically susceptible subjects the mucosal immune system displays an unbalanced response towards luminal antigens. Extensive research has well established that gut microbial flora is essential for the induction of IBD, but no specific microorganism has been convincingly associated with the pathogenesis. Permeability defects in the intestinal epithelium, as primary event or secondary to the inflammation, may play an important role in the onset or perpetuation of IBD. Although the exact mechanism of IBD is not known, most probable inducers are commensal bacteria and several immune and inflammatory mediators are thought to be involved ${ }^{[25-29]}$. Current methods of treatment vary from eliminating luminal bacteria by antibiotics (e.g. metronidazole, ciprofloxacin), over modulating one or more of these immune or inflammatory mediators (e.g. corticosteroids, infliximab, mesalazine), to immunosuppression (e.g. 6mercaptopurine). When antibiotics, antiinflammatories, immunomodulators or -suppressors do not help, surgical removal of the affected areas serves as a last resort. Bacterial resistance, undesirable side effects, high costs of production, in vivo instability, poor bioavailability and limited routes of administration are major drawbacks of the current treatment drugs.

In a first trial, PB6 was evaluated, in comparison to vancomycin, for its efficacy in a hamster model of clindamycin-induced CDAD, a well established and highly sensitive model of this infection ${ }^{[30]}$. Clindamycin induces in hamsters a lethal colitis that is accompanied by severe diarrhea and body weight loss. This colitis in hamsters is more extreme, but similar to antibioticassociated colitis in man.

In three other trials the efficacy of PB6 in treating TNBS-induced colitis in a rat model of IBD was determined and evaluated in comparison to the probiotic $S$. boulardii and the standard drugs mesalazine and infliximab. The TNBS-induced colitis model is a well established, reliable model that is being widely used to examine the efficiency of drugs aimed at treating $\mathrm{IBD}^{[31]}$.

\section{MATERIALS AND METHODS}

\section{Hamster model of clindamycin-induced CDAD}

Study design: The study was done on behalf of Kemin Pharma bvba at the test facility of Kemin Nutritional Technologies (Gummidipundi, India) and has complied with all relevant federal guidelines and institutional policies. 42 male Golden Syrian hamsters were obtained from the National Centre for Laboratory Animal Sciences, NIN (Hyderabad, India). At the beginning of the treatment period, the animals were 12 to 14 weeks old. Upon their arrival at the test facility, the animals were given a complete clinical examination under the supervision of a veterinarian to ensure that they were in good condition. The animals were 
acclimatized to the study conditions for a period of at least 7 days. Body weights were recorded before allocation of the animals into the study groups at the start of the trial. The animals were housed individually in polycarbonate cages $(290 \times 220 \times 140 \mathrm{~mm}, \mathrm{LxW} \times \mathrm{H})$. The animal room and test room conditions were set as follows: temperature: $22 \pm 4^{\circ} \mathrm{C}$, relative humidity: $50 \pm$ 20\%, light/dark cycle: $12 \mathrm{hr} / 12 \mathrm{hr}$ (light 07.00-19.00) and ventilation: approximately 7 cycles $\mathrm{hr}^{-1}$ of filtered, non-recycled air. All animals had free access to Hamster Pellet Feed (NIN, Hyderabad) and Aquaguard purified water ad libitum. Animals were allocated to one of 7 study groups (A to G). In groups A to E $C$. difficile associated diarrhea was induced by administering 10000 CFU's of toxigenic C. difficile ATCC 9689 orally on day 0, followed by a subcutaneous injection, in the trunk region just behind the ears, with clindamycin $100 \mathrm{mg} \mathrm{kg}^{-1}$ on day 1 . Group A received no further treatment. Group B was treated once daily from days 2 to 6 with vancomycin $50 \mathrm{mg}$ $\mathrm{kg}^{-1}$ by oral gavage. Groups $\mathrm{C}, \mathrm{D}$ and $\mathrm{E}$ were treated with PB6 at a dose of $1.5 \times 10^{9}$ (PB6 high), $1.5 \times 10^{7}$ (PB6 medium) and $1.5 \times 10^{6} \mathrm{CFU} \mathrm{kg}{ }^{-1}$ (PB6 low), 3 times daily (tid, ter in die) with $4 \mathrm{hrs}$ interdose (first dose at $9.30 \mathrm{am}$ ) from day 1 to 6 by oral gavage. On day 1 the first dose of PB6 was given $1 \mathrm{hr}$ after the injection of clindamycin. The treatments with PB6 were started 1 day earlier than the vancomycin treatment to allow for the transport of the bacterial cells, more than $90 \%$ of which were in spore form, to the colon and for their probable germination ${ }^{[32]}$ and production of antimicrobials. In groups $\mathrm{F}$ and $\mathrm{G}$ CDAD was not induced and the animals were given PB6 at a dose of $1.5 \times 10^{9} \mathrm{CFU} \mathrm{kg}{ }^{-1}$, tid with $4 \mathrm{hrs}$ interdose, from day 1 to 6 by oral gavage. Twice daily, up to day 15, observations were made for clinical signs and mortality. Signs of diarrhea were scored as being mild, moderate or severe. Body weights of animals were recorded on day 0,7 and 14. For groups $A$ to $E$ fecal samples were tested on days 1,2 and 7 for the presence of Clostridium toxin A and B using ImmunoCard Toxins A\&B (Meridian life sciences). On day 1 fecal samples were taken before clindamycin administration. On days 2 and 7 fecal samples were taken between the second and third treatment dose.

Study preparations: A Culti-loop (Oxoid, Basingstoke, England) containing the toxigenic $C$. difficile ATCC 9689 (type strain) was inoculated according to the manufacturer's instructions and the broth was diluted with saline to obtain $10000 \mathrm{CFU}$ $\mathrm{mL}^{-1}$. C. difficile ATCC 9689 is an $\mathrm{A}+\mathrm{B}+$ strain producing both toxins $\mathrm{A}$ and $\mathrm{B}$. Clindamycin hydrochloride (Pharmacia, Puurs, Belgium) and vancomycin (Neon Laboratories, Mumbai, India) were suspended in double distilled water to a concentration of 10 and $50 \mathrm{mg} \mathrm{mL}^{-1}$ respectively. Dose volumes were 10 and $1 \mathrm{~mL} \mathrm{~kg}^{-1}$ body weight for clindamycin and vancomycin respectively. PB6 Dry (Kemin Health, Des Moines, USA), a Bacillus subtilis 'PB6' fermentation broth dried on a malto- and cyclodextrin carrier, was suspended in double distilled water to concentrations of $1.5 \times 10^{8}, 1.5 \times 10^{6}$ or $1.5 \times 10^{5} \mathrm{CFU} \mathrm{mL}{ }^{-1}$. Dose volume was $10 \mathrm{~mL} \mathrm{~kg}^{-1}$ bodyweight. More than $90 \%$ of the viable cells in PB6 Dry were present as spores. Fresh preparations were made prior to each administration. Clindamycin, vancomycin and PB6 were administered on the basis of the last individual body weight measured. All preparations were stirred vigorously before each dosing.

\section{Rat model of IBD}

Study design: Three consecutive trials were done on behalf of Kemin Pharma at the test facility of Kemin Nutritional Technologies (Gummidipundi, India) in compliance with all federal government guidelines and institutional policies. The first trial was intended to search for a possible effect of PB6 on 2,4,6trinitrobenzenesulfonic acid (TNBS)-induced colitis in rats. The next two trials served to evaluate the effect found, in comparison with mesalazine and infliximab. In all 3 trials the day of induction of colitis was set as day 1 .

Male Wistar rats were obtained from the National Centre for Laboratory Animal Sciences (Hyderabad, India) (trial 1 and 2) or from the Department of Animal Medicine, TANUVAS (Chennai, India) (trial 3). At the beginning of the treatment period, the animals were 12 to 15 (trial 1) and 10 to 12 (trials 2 and 3) weeks old. Upon their arrival at the test facility, the animals were given a complete clinical examination under the supervision of a veterinarian to ensure that they were in good condition. The animals were acclimated to the study conditions for a period of at least 7 days. Per trial, animals were randomised and allocated to one of the study groups. Each group consisted of 5 animals. The animals were housed per study group in polycarbonate cages $(421 \times 290 \times 190 \mathrm{~mm}, \mathrm{LxWxH})$. The animal room and test room conditions were set as follows: temperature: $22 \pm 3{ }^{\circ} \mathrm{C}$, relative humidity: $50 \pm 20 \%$, light/dark cycle: $12 \mathrm{hr} / 12 \mathrm{hr}$ (light 07.00-19.00) and ventilation: approximately 7 cycles $\mathrm{hr}^{-1}$ of filtered, nonrecycled air. All animals had free access (except for the overnight fasting prior to TNBS administration) to rat pellet feed from the National Institute of Nutrition (NIN, Hyderabad, India) and Aquaguard purified water ad libitum. 
Colitis was induced, after an overnight fast, using a single intrarectal administration of TNBS at $100 \mathrm{mg}$ $\mathrm{kg}^{-1}$ body weight, $8 \mathrm{~cm}$ proximal to the anus. The colitis-negative control rats were given saline intrarectally ( $0.5 \mathrm{ml}$ per animal once) on day 1.Colitisnegative and colitis-positive control groups were given distilled water orally at $10 \mathrm{~mL} \mathrm{~kg}^{-1}$, tid with $4 \mathrm{~h}$ inter dosing, starting on day 1 and up to and including day 7 . In the first trial one group was given PB6 $\left(8 \times 10^{7} \mathrm{CFU}\right.$ $\mathrm{kg}^{-1}$ ), tid with $4 \mathrm{hrs}$ inter dosing, starting 7 days before induction of colitis and up to and including day 7. The other groups were treated with PB6 $\left(8 \times 10^{7}\right.$ or $1.5 \mathrm{x}$ $10^{9} \mathrm{CFU} \mathrm{kg}{ }^{-1}$ respectively), tid with 4 hrs inter dosing, starting on day 1 and up to and including day 7. In the second trial, groups with TNBS-induced colitis were treated with PB6 $\left(1.5 \times 10^{8}\right.$ or $1.5 \times 10^{9} \mathrm{CFU} \mathrm{kg}{ }^{-1}$ respectively), tid with $4 \mathrm{hrs}$ inter dosing, starting on day 1 and up to and including day 7; with mesalazine (250 mg.kg.day ${ }^{-1}$ ), starting on day 1 and up to and including day 7; or with infliximab $\left(3 \mathrm{mg} \mathrm{kg}^{-1}\right)$ as a single dose on day 1 . The third trial partially repeated the second one concerning the PB6 $\left(1.5 \times 10^{8} \mathrm{CFU} \mathrm{kg}{ }^{-1}\right)$, mesalazine and infliximab treatments and included an additional treatment with $S$. boulardii $\left(1.5 \times 10^{8} \mathrm{CFU}\right.$ $\mathrm{kg}^{-1}$ ), tid with $4 \mathrm{hrs}$ inter dosing, starting on day 1 and up to and including day 7. The first or only (in case of infliximab) dose of treatment was given within 2 (distilled water, PB6, S. boulardii and mesalazine) or 3 (infliximab) hrs after administration of TNBS. Except for infliximab, which was injected intraveneously, all treatments were administered by gavage. Twice daily observations were made for clinical signs and mortality. Body weights of animals were recorded on days $-7,4$ and 8 (trial 1), or days1, 4 and 7 (trials 2 and 3). Percent body weight gain was calculated based on the first and last recording. On day 8 animals were sacrificed and a 5 $\mathrm{cm}$ long segment of the colon (from 10 to $5 \mathrm{~cm}$ proximal to the anus) was excised. These segments were opened longitudinally. Contents were removed by washing with saline and gross morphology was scored using the following scale: 0 . no ulcers or inflammation, 1. no ulcers only local hyperaemia, 2. ulceration without hyperaemia, 3. ulceration and inflammation at one site only, 4. two or more sites of ulceration and inflammation and 5. ulceration extending more than 2 $\mathrm{cm}$. The weight of each $5 \mathrm{~cm}$ colonic segment was also recorded to assess inflammatory induced edema.

Study preparations: TNBS 5\% (w/v) in water (SigmaAldrich, St Louis, USA) was diluted to a $2.5 \%$ solution with ethanol $50 \%$. Dose volume was $4 \mathrm{~mL} \mathrm{~kg}^{-1}$ body weight. PB6 Dry (Kemin Health, Des Moines, USA), a Bacillus subtilis 'PB6' fermentation broth dried on a malto- and cyclodextrin carrier, was suspended in distilled water to a concentration of $8 \times 10^{6}, 1.5 \times 10^{7}$ or $1.5 \times 10^{8} \mathrm{CFU} \mathrm{mL}{ }^{-1}$. Dose volume was $10 \mathrm{~mL} \mathrm{~kg}^{-1}$ body weight. S. boulardii (Enterol ${ }^{\circledR}$, Biodiphar, Brussel, Belgium) was suspended in distilled water to a concentration of $1.5 \times 10^{7} \mathrm{CFU} \mathrm{mL} \mathrm{m}^{-1}$. Dose volume was $10 \mathrm{~mL} \mathrm{~kg}^{-1}$ body weight. Mesalazine (5-aminosalicylic acid) $\quad\left(\right.$ Mesacol $^{\circledR}$, Sun Pharmaceutical Ind. Ltd, Mumbai, India) tablets were powdered using pestle and mortar and a solution in distilled water was prepared containing $25 \mathrm{mg}$ 5-aminosalicylic acid per ml. Dose volume was $10 \mathrm{~mL} \mathrm{~kg}^{-1}$ body weight. Infliximab (Remicade ${ }^{\circledR}$, Centocor B.V., Leiden, The Netherlands) was first reconstituted with $10 \mathrm{ml}$ water for injection and was further diluted to $2 \mathrm{mg} \mathrm{mL} \mathrm{m}^{-1}$ concentration using sterile saline. Dose volume employed was $1.5 \mathrm{~mL}$ $\mathrm{kg}^{-1}$ body weight. All body weight dependant doses were administered on the basis of the last individual body weight taken. Fresh preparations were made prior to each administration. The preparations were stirred vigorously before each dosing.

\section{RESULTS}

\section{CDAD hamster trial}

Effect of PB6 on $C$. difficile-associated diarrhea: About 6 hrs after clindamycin was administered mild diarrhea was observed in 3 animals of the no treatment group (A) and in 1 animal of the group to be treated with vancomycin (B). In the groups $\mathrm{C}, \mathrm{D}$ and $\mathrm{E}$, in which the animals received their first dose of PB6 about $1 \mathrm{hr}$ after the administration of clindamycin, none of the animals showed any sign of diarrhea that same day. The number of animals suffering from diarrhea and the severity of diarrhea evolved differently between treatment groups the following 2 days. All groups in which CDAD was induced, showed signs of diarrhea. In groups $\mathrm{F}$ and $\mathrm{G}$, without induction of $\mathrm{CDAD}$, no diarrhea was observed. On day 3 the number of animals with diarrhea was 6, 2, 4, 6 and 6 in groups A to E. The intensity of diarrhea was less in the group treated with vancomycin and the group treated with the high dose of PB6. In the no treatment group as well as in the low and mid dose PB6 groups the stool was very watery and whole the abdominal area was wet.

Effect of PB6 on $C$. difficile-associated mortality: At the end of day 3 all animals of groups in which CDAD was induced were still alive but several of them were showing signs of severe diarrhea. On day 4 , three days after clindamycin administration, the first animals died. 
Am. J. Infect. Dis., 3 (4): 255-266, 2007

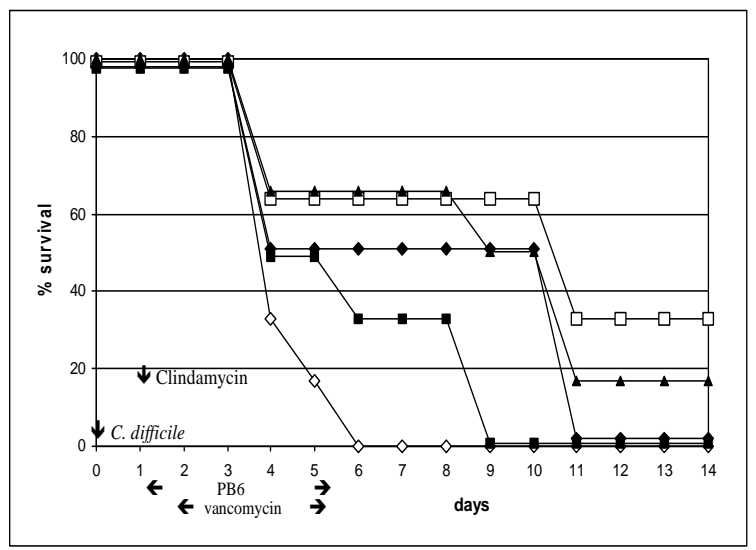

Fig. 1: $\quad$ Percent survival in groups in which CDAD was induced. (No treatment $\diamond$, vancomycin $\square$, PB6 high $\Delta$, PB6 medium $\bullet$, PB6 low $\square$ )

At the end of the treatment period, on day 6, all hamsters in the no treatment group had died. Survival was highest in the vancomycin and PB6 high dose treatment groups, where 4 out of 6 had survived (Fig. 1). In groups $F$ and $G$, treated with a high dose of PB6 without prior induction of CDAD, no animals died.

Effect of PB6 on $C$. difficile-associated weight loss: On day 7 a decreased average body weight was observed in all groups in which CDAD was induced. There was a clear inverse dose response relation with PB6 concerning this decrease in weight (Table 1). The animals receiving a low dose of PB6, on average lost 3 times more weight than the animals which received a high dose. Weight loss was least with vancomycin treatment. The average body weight in the two groups treated with a high dose of PB6 in which CDAD was not induced, had slightly increased over the same time period.

C. difficile toxins in fecal samples: The presence of Clostridium toxin A and B in fecal samples of the animals in which CDAD was induced, was checked on days 1, 2 and 7. As expected, groups showing high mortality and a high decrease of average body weight also had a higher percentage of animals tested positive for these toxins. The number of animals tested positive in groups $A$ to E respectively $(n=6)$ was on day $1: 3,0$, $0,0,0$ and on day 2: 5, 2, 3, 3, 5. On day 7 all animals in group A had died and in groups B to E 1, 2, 2, 2 animals out of $4,4,3,2$ survivors tested positive respectively.

Post-treatment effect of PB6: Last day of treatment was day 6. In all groups in which CDAD was induced, more animals surviving on day 6 died post-treatment. All animals of the PB6 low dose treatment group were dead at day 9, those of the PB6 medium dose treatment group at day 11. Vancomycin and PB6 high dose, with respectively 2 and 1 survivor, were the only treatments that prevented a $100 \%$ mortality at day 14 (Fig. 1).

\section{IBD rat trials}

Trial 1: Shortly after induction of colitis with TNBS several animals showed signs of diarrhea. The number of observations of diarrhea recorded (and the number of animals affected) in the different treatment groups from day 1 up to and including day 7 were 5 (2), 38 (5), 21 (3), 26 (4) and 12 (3) for colitis-negative control, colitis-positive control, PB6 $8 \times 10^{7} \mathrm{CFU} \mathrm{kg}^{-1}$ tid preand post-induction treatment, PB6 $8 \times 10^{7} \mathrm{CFU} \mathrm{kg}^{-1}$ tid post-induction treatment and PB6 $1.5 \times 10^{9} \mathrm{CFU} \mathrm{kg}^{-1}$ tid post-induction treatment respectively. The colitis and accompanying diarrhea, in some cases probably tempered by the treatment, also reflected in the groups mean body weight and body weight gain data (Table 2).

Due to the administration of TNBS, preceded by an overnight fasting and to the onset of colitis, body weight gain retarded or animals even started losing weight. On days 4 and 8 after induction of colitis, statistically significant differences in mean body weight were observed between groups. At the end of the treatment period, only the colitis-negative control group and the group treated for 7 days post-induction with 1.5

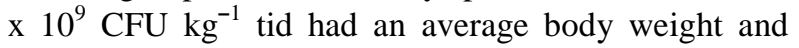
body weight gain that was clearly higher than the colitis-positive control group $(\mathrm{P}<0.05)$ (Table 2).

Statistically significant differences were also observed between groups in score for gross morphology of the intestinal wall of a $5 \mathrm{~cm}$ colon segment and in the wet weights of these segments (Table 3 ). The only groups that could be clearly distinguished from the colitis-positive control group $(\mathrm{P}<0.05)$ were again the colitis-negative control group and the group treated with PB6 $1.5 \times 10^{9} \mathrm{CFU} \mathrm{kg}^{-1}$ tid. All other groups showed an increased colon segment weight (as a measure of inflammatory edema) and a clear ulceration of the colon wall comparable to that of the colitispositive control group. The macroscopical colon wall health status of the group treated with PB6 $1.5 \times 10^{9}$ CFU kg ${ }^{-1}$ tid for 7 days was comparable to that of the colitis-negative control group. PB6 at a dose of $8 \times 10^{7}$ CFU $\mathrm{kg}^{-1}$ tid for 7 days was possibly close to the minimal dose effective to suppress colitis as induced by TNBS in rats in this trial. Some of the animals in this group had clear signs of colitis while in others these 
Am. J. Infect. Dis., 3 (4): 255-266, 2007

Table 1: The effect of treatment on body weight and body weight gain in male Golden Syrian hamsters with clindamycin-induced $C$. difficile associated diarrhea

\begin{tabular}{|c|c|c|c|}
\hline \multirow[t]{2}{*}{ Treatment } & \multicolumn{2}{|l|}{ Body weight (g) } & \multirow{2}{*}{$\begin{array}{l}\text { Body weight gain }(\%) \\
\text { Day } 0 \text { to } 7\end{array}$} \\
\hline & Day 0 & Day 7 & \\
\hline \multicolumn{4}{|l|}{ CDAD Induced } \\
\hline No treatment & $219.0 \pm 5.1^{\mathrm{a}}(\mathrm{n}=6)$ & $\mathrm{q}$ & $\mathrm{q}$ \\
\hline Vancomycin & $218.0 \pm 4.6^{a}(\mathrm{n}=6)$ & $215.8 \pm 1.7^{\mathrm{ab}}(\mathrm{n}=4)$ & $-1.7 \pm 1.8^{\mathrm{bc}}$ \\
\hline PB6 $1.5 \times 10^{9} \mathrm{CFU} \mathrm{kg}{ }^{-1}$ tid & $218.8 \pm 4.0^{\mathrm{a}}(\mathrm{n}=6)$ & $206.5 \pm 5.7^{\mathrm{ab}}(\mathrm{n}=4)$ & $-5.0 \pm 2.1^{c}$ \\
\hline PB6 $1.5 \times 10^{7} \mathrm{CFU} \mathrm{kg}{ }^{-1}$ tid & $217.8 \pm 3.2^{a}(\mathrm{n}=6)$ & $189.0 \pm 2.7^{\mathrm{b}}(\mathrm{n}=3)$ & $-12.9 \pm 0.7^{\mathrm{d}}$ \\
\hline $\begin{array}{l}\text { PB6 } 1.5 \times 10^{6} \mathrm{CFU} \mathrm{kg}^{-1} \text { tid } \\
\text { No CDAD induced }\end{array}$ & $217.2 \pm 4.5^{a}(n=6)$ & $176.5 \pm 2.1^{\mathrm{b}}(\mathrm{n}=2)$ & $-17.9 \pm 1.2^{\mathrm{d}}$ \\
\hline PB6 $1.5 \times 10^{9} \mathrm{CFU} \mathrm{kg}{ }^{-1}$ tid & $217.8 \pm 2.3^{a}(n=6)$ & $230.0 \pm 7.6^{\mathrm{a}}(\mathrm{n}=6)$ & $5.6 \pm 3.1^{\mathrm{a}}$ \\
\hline PB6 $1.5 \times 10^{9} \mathrm{CFU} \mathrm{kg}{ }^{-1}$ tid & $219.2 \pm 3.9^{\text {a }}(\mathrm{n}=6)$ & $225.8 \pm 4.8^{a b}(n=6)$ & $3.1 \pm 3.2^{\mathrm{ab}}$ \\
\hline
\end{tabular}

Table 2: The effect of treatment on body weight and body weight gain in male Wistar rats with TNBS-induced colitis. Trial 1.

\begin{tabular}{|c|c|c|c|c|}
\hline \multirow[b]{2}{*}{ Treatment } & \multicolumn{3}{|c|}{ Body weight (g) } & \multirow[b]{2}{*}{ Body weight gain (\%) } \\
\hline & Day -7 & Day 4 & Day 8 & \\
\hline Colitis-negative control & $253 \pm 15$ & $287 \pm 5^{*}$ & $288 \pm 8^{*}$ & $14.1 \pm 5.2^{*}$ \\
\hline Colitis-positive control & $252 \pm 16$ & $238 \pm 14$ & $237 \pm 13$ & $-5.7 \pm 5.3$ \\
\hline PB6 $8 \times 10^{7} \mathrm{CFU} \mathrm{kg}{ }^{-1}$ tid & & & & \\
\hline (pre- and post-induction) & $254 \pm 12$ & $257 \pm 2$ & $262 \pm 9$ & $3.1 \pm 7.7$ \\
\hline PB6 $8 \times 10^{7} \mathrm{CFU} \mathrm{kg}^{-1}$ tid & $256 \pm 11$ & $248 \pm 19$ & $248 \pm 31$ & $-3.1 \pm 11.7$ \\
\hline PB6 $1.5 \times 10^{9} \mathrm{CFU} \mathrm{kg}^{-1}$ tid & $255 \pm 8$ & $263 \pm 11^{*}$ & $277 \pm 10^{*}$ & $8.4 \pm 1.8^{*}$ \\
\hline
\end{tabular}

Data are expressed as mean \pm SD. The day of induction of colitis is defined as day 1. PB6 was administered for 7 days post-induction. One group received PB6 also prophylactically for 7 days pre-induction. ${ }^{*}$ Significantly different from the positive control group (Dunnett, $\left.\mathrm{P}<0.05\right)$. tid, ter in die (three times a day).

Table 3: The effect of treatment on the gross morphology score for the colon wall and the wet weight of a $5 \mathrm{~cm}$ colon segment in male Wistar rats with TNBS-induced colitis. Trial 1.

\begin{tabular}{lll}
\hline Treatment & Gross morphology score & Wet weight $(\mathrm{g})$ \\
\hline Colitis-negative control & $0.2 \pm 0.4^{*}$ & $0.465 \pm 0.147^{*}$ \\
Colitis-positive control & $3.2 \pm 1.1$ & $0.858 \pm 0.152$ \\
PB6 8 x 10 $\mathrm{CFU} \mathrm{kg}$ tid & & \\
(pre- and post-induction) & $2.0 \pm 1.4$ & $0.692 \pm 0.135$ \\
PB6 8 $10^{7} \mathrm{CFU} \mathrm{kg}{ }^{-1}$ tid & $2.4 \pm 1.3$ & $0.745 \pm 0.231$ \\
PB6 $1.5 \times 10^{9} \mathrm{CFU} \mathrm{kg}^{-1}$ tid & $0.6 \pm 0.5^{*}$ & $0.504 \pm 0.172^{*}$ \\
\hline
\end{tabular}

Data are expressed as mean \pm SD. The day of induction of colitis is defined as day 1. PB6 was administered for 7 days post-induction. One group received PB6 also prophylactically for 7 days pre-induction. ${ }^{*}$ Significantly different from the positive control group (Dunnett, $\left.\mathrm{P}<0.05\right)$. tid, ter in die (three times a day).

were almost absent. This was also the case in the group treated both pre- and post-induction with that same dose. An additional prophylactic treatment with PB6 8 x $10^{7} \mathrm{CFU} \mathrm{kg}{ }^{-1}$ tid for 7 days did not improve the results of a 7 days treatment post-induction.

Trials 2 and 3: In the second trial one of the rats treated with infliximab died on day 2 . This animal had been given a second injection on day 1 because at the first one drug solution oozed out. Also in these trials some animals showed mild signs of diarrhea after induction of colitis with TNBS. The number of observations of diarrhea recorded (and the number of animals affected) in the different treatment groups from day 1 up to and including day 7 were in the second trial 0, 22 (2), 4 (1), 0 (0), 0 (0) and 8 (1) for colitis-negative control, colitis-positive control, PB6 $1.5 \times 10^{8} \mathrm{CFU}$ $\mathrm{kg}^{-1}$ tid, PB6 $1.5 \times 10^{9} \mathrm{CFU} \mathrm{kg}{ }^{-1}$ tid, mesalazine 250 mg.kg.day ${ }^{-1}$ and infliximab $3 \mathrm{mg} \mathrm{kg}^{-1}$ single dose respectively; and in the third trial 0, 42 (4), 10 (1), 34 (3), 12 (1) and 24 (2) for colitis-negative control, colitis-positive control, PB6 $1.5 \times 10^{8} \mathrm{CFU} \mathrm{kg}{ }^{-1}$ tid,.S. boulardii $1.5 \times 10^{8} \mathrm{CFU} \mathrm{kg}^{-1}$ tid, mesalazine 250 mg.kg.day ${ }^{-1}$ and infliximab $3 \mathrm{mg} \mathrm{kg}{ }^{-1}$ single dose respectively. 


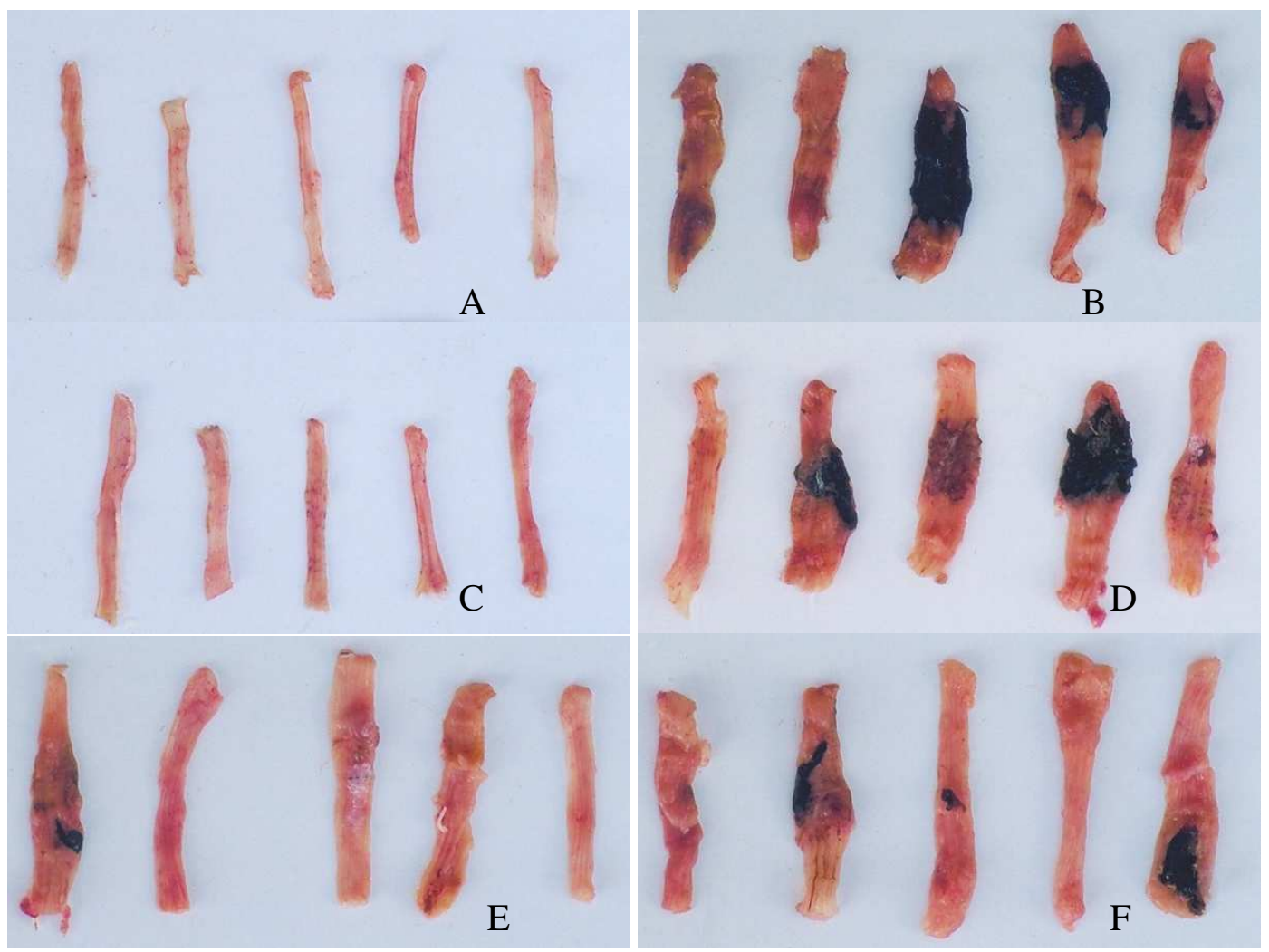

Fig. 2: Longitudinally opened colon segments of the colitis-negative control group (A), the colitis-positive control group (B) and of the groups treated for 7 days post-induction with PB6 $\left(1.510^{8} \mathrm{CFU} \mathrm{kg}^{-1}\right.$ tid) (C), S. boulardii $\left(1.510^{8} \mathrm{CFU} \mathrm{kg}{ }^{-1}\right.$ tid) (D), or mesalazine $\left(250 \mathrm{mg} \cdot \mathrm{kg} \mathrm{day}{ }^{-1}\right)(\mathrm{E})$ or treated with a single dose of infliximab $\left(3 \mathrm{mg} \mathrm{kg}^{-1}\right)$ (F) post-induction on day 1. Photographs of trial 3 colon segments

Table 4: The effect of treatment on body weight and body weight gain in male Wistar rats with TNBS-induced colitis. Trials 2 and 3. Body weight $(\mathrm{g})$

\begin{tabular}{|c|c|c|c|c|}
\hline Treatment & Day 1 & Day 4 & Day 7 & Body weight gain $(\%)$ \\
\hline \multicolumn{5}{|l|}{ Trial 2} \\
\hline Colitis-negative control & $178 \pm 17$ & $191 \pm 13^{*}$ & $203 \pm 13^{*}$ & $14.3 \pm 4.5^{*}$ \\
\hline Colitis-positive control & $182 \pm 15$ & $167 \pm 16$ & $159 \pm 18$ & $-13.0 \pm 4.5$ \\
\hline PB6 $1.5 \times 10^{8} \mathrm{CFU} \mathrm{kg}^{-1}$ tid & $180 \pm 13$ & $186 \pm 13$ & $195 \pm 10$ * & $8.8 \pm 8.0^{*}$ \\
\hline PB6 $1.5 \times 10^{9} \mathrm{CFU} \mathrm{kg}^{-1}$ tid & $180 \pm 16$ & $187 \pm 15$ & $199 \pm 17^{*}$ & $10.3 \pm 1.1^{*}$ \\
\hline Mesalazine $250 \mathrm{mg} \mathrm{kg}^{-1}$ & $181 \pm 11$ & $187 \pm 10$ & $196 \pm 10^{*}$ & $8.8 \pm 1.4^{*}$ \\
\hline Infliximab $3 \mathrm{mg} \mathrm{kg}^{-1}$ & $180 \pm 14$ & $183 \pm 7$ & $186 \pm 6^{*}$ & $1.3 \pm 5.4^{*}$ \\
\hline \multicolumn{5}{|l|}{ Trial 3} \\
\hline Colitis-negative control & $207 \pm 20$ & $210 \pm 19$ & $214 \pm 17$ & $3.5 \pm 1.8^{*}$ \\
\hline Colitis-positive control & $208 \pm 21$ & $196 \pm 21$ & $193 \pm 19$ & $-7.5 \pm 3.2$ \\
\hline PB6 $1.5 \times 10^{8} \mathrm{CFU} \mathrm{kg}^{-1}$ tid & $206 \pm 23$ & $206 \pm 23$ & $212 \pm 26$ & $2.9 \pm 3.1^{*}$ \\
\hline S. boulardii $1.5 \times 10^{8} \mathrm{CFU} \mathrm{kg}{ }^{-1}$ tid & $207 \pm 17$ & $196 \pm 16$ & $195 \pm 24$ & $-5.7 \pm 5.6$ \\
\hline Mesalazine $250 \mathrm{mg} \mathrm{kg}^{-1}$ & $207 \pm 12$ & $199 \pm 18$ & $201 \pm 20$ & $-3.3 \pm 5.5$ \\
\hline Infliximab $3 \mathrm{mg} \mathrm{kg}^{-1}$ & $208 \pm 15$ & $197 \pm 14$ & $197 \pm 13$ & $-5.2 \pm 3.4$ \\
\hline
\end{tabular}

Data are expressed as mean \pm SD. The day of induction of colitis is defined as day 1. PB6, S. boulardii and mesalazine were administered for 7 days post-induction. Infliximab was administered on day 1 only. ${ }^{*}$ Significantly different from the positive control group (Dunnett, $\left.\mathrm{P}<0.05\right)$. tid, ter in die (three times a day). 
Am. J. Infect. Dis., 3 (4): 255-266, 2007

Table 5: The effect of treatment on the gross morphology score for the colon wall and the wet weight of a $5 \mathrm{~cm}$ colon segment in male Wistar rats with TNBS-induced colitis. Trials 2 and 3 .

\begin{tabular}{|c|c|c|}
\hline Treatment & Gross morphology score & Wet weight $(\mathrm{g})$ \\
\hline \multicolumn{3}{|l|}{ Trial 2} \\
\hline Colitis-negative control & $0.2 \pm 0.4^{*}$ & $0.378 \pm 0.047^{*}$ \\
\hline Colitis-positive control & $3.8 \pm 0.8$ & $1.406 \pm 0.209$ \\
\hline PB6 $1.5 \times 10^{8} \mathrm{CFU} \mathrm{kg}{ }^{-1}$ tid & $1.0 \pm 0.0^{*}$ & $0.443 \pm 0.063^{*}$ \\
\hline PB6 $1.5 \times 10^{9} \mathrm{CFU} \mathrm{kg}{ }^{-1}$ tid & $0.6 \pm 0.5^{*}$ & $0.513 \pm 0.317^{*}$ \\
\hline Mesalazine $250 \mathrm{mg} \mathrm{kg}^{-1}$ & $0.8 \pm 0.4^{*}$ & $0.435 \pm 0.052^{*}$ \\
\hline Infliximab $3 \mathrm{mg} \mathrm{kg}^{-1}$ & $3.0 \pm 1.4$ & $1.055 \pm 0.490$ \\
\hline \multicolumn{3}{|l|}{ Trial 3} \\
\hline Colitis-negative control & $0.0 \pm 0.0^{*}$ & $0.274 \pm 0.049^{*}$ \\
\hline Colitis-positive control & $3.4 \pm 1.1$ & $0.832 \pm 0.216$ \\
\hline PB6 $1.5 \times 10^{8} \mathrm{CFU} \mathrm{kg}^{-1}$ tid & $0.6 \pm 0.5^{*}$ & $0.280 \pm 0.039^{*}$ \\
\hline S. boulardii $1.5 \times 10^{8} \mathrm{CFU} \mathrm{kg}^{-1}$ tid & $2.8 \pm 1.3$ & $0.735 \pm 0.221$ \\
\hline Mesalazine $250 \mathrm{mg} \mathrm{kg}^{-1}$ & $1.2 \pm 0.8^{*}$ & $0.445 \pm 0.145^{*}$ \\
\hline Infliximab $3 \mathrm{mg} \mathrm{kg}^{-1}$ & $2.0 \pm 0.7$ & $0.657 \pm 0.076$ \\
\hline
\end{tabular}

Data are expressed as mean \pm SD. The day of induction of colitis is defined as day 1. PB6, S. boulardii and mesalazine were administered for 7 days post-induction. Infliximab was administered on day 1 only. "Significantly different from the positive control group (Dunnett, $\mathrm{P}<0.05)$. tid, ter in die (three times a day).

The colitis-positive control groups showed substantial body weight loss accompanying the colitis. In trial 2 this loss was higher than in trial 3, what may be related to a difference in genetic background and a possible difference in age and growth rate at the moment of induction of colitis. In trial 2 there was a lower average body weight in general at the start and a higher body weight gain in terms of percentage over the trial period of the colitis-negative control group. While common treatments in trial 2 all significantly suppressed the negative effect of colitis on body weight gain, in trial 3 this was only the case with PB6 treatment (Table 4).

The greater impact of colitis on the animals in trial 2 probably left more room for improvement by any of the treatments. PB6 and mesalazine always resulted in a colon segment weight and a gross morphology score for the colon wall that could be clearly distincted from those of the colitis-positive control group (Table 5).

The health status of the colon wall in rats with TNBS-induced colitis treated with PB6 and mesalazine was macroscopically the same as that in colitis-free rats. For an unknown reason mesalazine treatment was somewhat less effective in trial 3, resulting in some ulcerations. Macroscopic pictures of the longitudinally opened colon segments clearly show the ulcerations and areas of necrotic tissue present in the positive control, the $S$. boulardii and the infliximab treatment groups and the absence thereof in the PB6 group (Fig. 2).

Common treatments in trial 2 resulted in colon segments with the same macroscopical appearance as in trial 3. At the end of the treatment period the average body weight gain and colon segment weight of rats treated with infliximab were intermediary to those of the colitis-negative and the colitis-positive control groups in both trials. This probably indicates that there was some effect of infliximab, although not statistically significant in these trials. In the colon segments of rats treated with PB6 there were no ulcerations. Only hyperaemia was observed in the majority of these segments. PB6 clearly attenuates inflammation in the rat colon wall as induced by intrarectal administration of TNBS. The efficacy of a 7 days post-induction treatment with $1.5 \times 10^{9} \mathrm{CFU} \mathrm{kg}^{-1}$ tid observed in trial 1 was confirmed in trial 2. While a 7 days postinduction treatment with PB6 $8 \times 10^{7} \mathrm{CFU} \mathrm{kg}^{-1}$ tid from the first trial was concluded to be ineffective, an increase of the CFU kg-1 tid to $1.5 \times 10^{8}$ proved in both following trials to be sufficient to reduce inflammation to an extent that the data of this treatment could no longer be statistically distinguished from those of the colitis-negative control group.

\section{DISCUSSION}

CDAD hamster trial: Vancomycin and in first place metronidazole are the drugs of choice for the treatment of $C$. difficile infection. Trials have repeatedly indicated that in hamsters death from clindamycin-induced colitis can be delayed by either of these two as long as they are administered. Death almost invariably occurs however after treatment is stopped ${ }^{[34-37]}$. Also in this trial, at the end of the observation period and 8 days after the end of the treatment, half of the surviving animals treated with vancomycin had died. Vancomycin and metronidazole fail to correct the predisposing conditions in the gut and being antibiotics, might even aggravate disruption of the intestinal flora which could account for the relapse of colitis in the hamster model and in humans once the antibiotic treatment is stopped. Agents that help to maintain or are able to restore the 
microbiological equilibrium within the intestinal tract may prevent proliferation of $C$. difficile and the occurrence of CDAD. The prophylactic use of probiotics in humans to help prevent possible diarrhea and other gastrointestinal disturbances associated with antibiotic treatment is well established and may constitute a valuable treatment support ${ }^{[38,39]}$. Studies conducted to date on a possible protective or therapeutic effect of probiotics against CDAD in humans however provide insufficient evidence for their routine clinical use with this infection ${ }^{[40]}$. Studies on the efficiency of probiotics in an animal model of CDAD are very scanty. Colonization with nontoxigenic $C$. difficile strains has proven to be highly effective in preventing CDAD in hamsters ${ }^{[41]}$. Although $C$. difficile is usually not associated with probiotics, the results obtained suggest that use of a probiotic strategy for CDAD prevention might be beneficial. The probiotic yeast Saccharomyces boulardii at 8 x $10^{10}$ CFU.kg.day ${ }^{-1}$, as a $5 \%$ suspension of a lyophilized powder in drinking water, has been shown to help prevent extensive overgrowth of $C$. difficile and to reduce clindamycin-induced cumulative mortality in hamsters by 10 to $35 \%$ when consumption of this yeast started prophylactically during at least 3 days before clindamycin exposure. Clindamycin doses exceeding $0.6 \mathrm{mg} \mathrm{kg}^{-1}$ however obliterated the protective effect ${ }^{[42]}$. In current trial a hundredfold higher, single subcutaneous dose of clindamycin $\left(100 \mathrm{mg} \mathrm{kg}^{-1}\right), 24 \mathrm{hr}$ after a single oral administration of $10^{4} \mathrm{CFU}$ of toxigenic $C$. difficile ATCC 9689, was used to induce CDAD in hamsters. The difference in the number of animals that tested positive for $C$. difficile toxins observed at the end of day 1 between the no treatment and the vancomycin groups, might seem to indicate that induction of CDAD failed in the latter. The onset of diarrhea in this group at the end of day 1 , the presence of toxin positives on day 2 and the further trial course is convincing of the opposite. Hamsters that were not treated after induction of CDAD this way all got severe diarrhea, lost weight and died within 3 to 5 days. The administration of PB6 in this trial started on the same day as the administration of clindamycin and not several days before, as was the case with $S$. boulardii in the aforementioned trial. Additionally, the fact that PB6 is susceptible to clindamycin might have impaired the effect of the initial doses. Nonetheless PB6 $\left(1.5 \times 10^{9}\right.$ $\mathrm{CFU} \mathrm{kg}{ }^{-1}$ tid for 6 days) reduced cumulative mortality over the treatment period by $66 \%$, to the same level as vancomycin (Fig. 1). A strong indication for the therapeutic potential of PB6 is the inverse dose response relation observed with $C$. difficile-associated weight loss. Animals receiving the low dose of PB6
(1.5 $\times 10^{6} \mathrm{CFU} \mathrm{\textrm {kg } ^ { - 1 }}$ tid) suffered most from diarrhea and lost the most weight. The weight loss with animals receiving the high dose $\left(1.5 \times 10^{9} \mathrm{CFU} \mathrm{kg}{ }^{-1}\right.$ tid $)$ was much less distinct and almost at the same level as with vancomycin treatment (Table 1). Under the conditions used in this trial, treatment with PB6 three times daily at $1.5 \times 10^{9} \mathrm{CFU} \mathrm{kg}{ }^{-1}$ for 6 days could not prevent further animal deaths after cessation of treatment (Fig. 1). The hamster model however represents an extreme form of CDAD of which the counterpart in humans, in most instances, is a milder condition.

The lack of knowledge on their mode(s) of action or the fact that these are at least not well defined, is something most probiotics have in common. Lactic acid bacteria, so far, are the best-documented probiotics. In contrast, the mechanisms responsible for the beneficial effect of other probiotics, especially the Bacillus species, have remained relatively unexplored. Additional experiments are needed to provide insight into the mechanism(s) responsible for the probiotic effect of PB6. At the moment we can only hypothesize based on the data we have. The production of surfactins active against Clostridium certainly could be an element in it. The biological activity of surfactins is supposedly related to its ability to destabilize and permeabilize membranes at very low concentrations, leading to membrane disintegration at higher concentrations. Micrographs from transmission electron microscopy have shown that when $C$. perfringens was incubated in vitro with PB6 culture supernatans centrifuged at $14000 \mathrm{~g}$ for $15 \mathrm{~min}$ and filter-sterilized, the cell wall of the bacteria was disrupted and cytoplasmic contents leaked into the exterior (Fig. 3). Disruption of viral lipid membranes and mycoplasma membranes by surfactin also has been observed by electron microscopy ${ }^{[16,17]}$.

Another possible mechanism is immunomodulation. As described in this article, PB6 is very effective in reducing the severeness of inflammation in trinitrobenzenesulfonic acid (TNBS)induced colitis in rats. This may be due to the putative anti-inflammatory activity of surfactin but may also be an indication that PB6 has immunomodulatory properties $^{[19]}$.

In summary, our results indicate that in hamsters treatment of clindamycin-induced CDAD with PB6 resulted in a dose related response. Diarrhea, body weight reduction and mortality were least with the highest dose of PB6. At treatment stop, the highest dose of PB6 showed to have been equally efficient as vancomycin in helping hamsters to survive clindamycin-induced CDAD. 


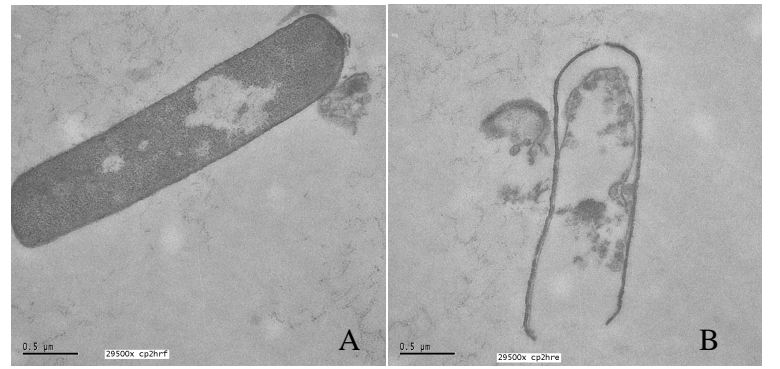

Fig. 3: The effect of PB6 (ATCC - PTA 6737) culture supernatant on Clostridium perfringens at $37^{\circ} \mathrm{C}$. (A) disruption of cell wall and loss of cytoplasmic contents into the exterior after 1 hr; (B) rupture and death of cell after 4 hrs (transmission electron micrograph: X 29500)

IBD rat trials: Studies conducted with animal models of colitis demonstrate that probiotic treatment can sometimes reduce the development of colitis and treat or attenuate established disease. Immunomodulation, improvement of epithelial barrier function, competitive exclusion of gastrointestinal pathogens and secretion of antimicrobial compounds which suppress the growth of harmful enteric bacteria, are all basic mechanisms by which probiotics can exert an effect. Overall studies in animal models indicate that various probiotic species have different effects in selected hosts and inflammatory conditions, linked to the likely involvement of multiple mechanisms of action ${ }^{[43-50]}$. In the current study Bacillus subtilis 'PB6' and the standard drug mesalazine showed to be very efficient in suppressing TNBS-induced colitis in a rat model of IBD. Mesalazine has in vitro and in vivo pharmacologic effects that decrease leukotriene production, scavenge for free radicals and inhibit leukocyte chemotaxis. While its exact mode of action is currently unknown, any of these biochemical mechanisms may play a role in the clinical effectiveness of mesalazine. Infliximab, the other standard drug we used as a reference in our evaluation, is a genetically engineered hybrid monoclonal antibody manufactured using cells containing human and mouse antibody genes. The drug works by binding to TNF- $\alpha$, a promotor of inflammation and essentially removing it from circulation. Our results with infliximab are comparable to those of a similar trial in rats where infliximab was administered at the same single dose of $3 \mathrm{mg} \mathrm{kg}^{-1}$ i.v. 2 days before induction of colitis with TNBS. In that trial, 8 days after induction, infliximab was shown to have some effect on body weight gain and colon gross morphology but not to reduce the level of edema significantly below that of the colitis-positive control group $^{[51]}$. For the anti-inflammatory effect observed with the oral administration of PB6 we hypothesize that this may be due to the probable germination of PB6 spores in the intestines, followed by bacterial growth and production of surfactins ${ }^{[32,52]}$. The demonstrated suppression of antiinflammatory responses by surfactin possibly relates to the selective inhibition of cytosolic
$\mathrm{PLA}_{2}$, which is known to contribute in the biosynthesis of several inflammatory mediators ${ }^{[19,53]}$. By its action on membrane phospholipids PLA $_{2}$ releases arachidonic acid and lysophospholipids; precursors of proinflammatory mediators, such as prostaglandins, thromboxanes, leukotrienes and platelet-activating factor. Diverse of these have been found to be strong mediators of intestinal inflammation ${ }^{[54]}$. Arachidonic acid also upregulates P38 mitogen-activated protein kinase (MAPK)-induced production of TNF- $\alpha$ and associated proinflammatory mediators ${ }^{[55]}$. Inhibition of cytosolic $\mathrm{PLA}_{2}$ therefore suppresses both the release of precursors of proinflammatory mediators and the upregulation of the P38 MAPK-induced production of proinflammatory mediators. Inhibitors of $\mathrm{PLA}_{2}$, such as quinacrine, p-bromophenacyl bromide and carboxymethylcellulose-linked phosphatidylethanolamine have been shown to attenuate intestinal inflammation in chemically induced and immunologically mediated animal models of $\operatorname{IBD}^{[56,57]}$. Surfactins have shown their antiinflammatory activity in different animal models. They ameliorated both tetradecanoyl phorbol-acetate- and acetic acid-induced mouse ear edema, carrageenan-induced rat paw edema and showed a potent dose dependant analgesic effect in the acetic acid-induced abdominal constriction assay in mice when administered intraperitoneally. In the latter test surfactins outperformed indomethacin, a common non-steroidal antiinflammatory drug, by $10-$ fold $^{[19]}$. Taking into account firstly the literature findings on the pivotal role of $\mathrm{PLA}_{2}$ in the inflammation process, on the suppression of inflammation by $\mathrm{PLA}_{2}$ inhibitors and on the inhibition of $\mathrm{PLA}_{2}$ by surfactin and secondly the production of the latter by $\mathrm{PB} 6$, it can be reasonably hypothesised that PB6 in the intestine mediates inhibition of $\mathrm{PLA}_{2}$ through the production of surfactins, thus reducing the deteriorating effects of TNBSinduced inflammation. Future studies, focusing on the fate of PB6 in the intestines, the possible production of surfactins in situ and the effect of PB6 on the levels of proinflammatory mediators, should verify this hypothesis. In humans, clinical trials suggest that selected probiotic species can prevent recurrent intestinal inflammation and possibly treat active IBD. The most convincing results to date are obtained with VSL\#3 in maintaining remission in recurrent or refractory pouchitis ${ }^{[23,589}$. VSL\#3 is a probiotic-mixture, consisting of four strains of Lactobacillus, three strains of Bifidobacterium and one strain of Streptococcus. VSL\#3 decreases the level of proinflammatory cytokines and the activity of inducible nitric oxide synthase and matrix metalloproteinase ${ }^{[59]}$. Its actual mechanism of action and whether all eight bacteria are required for its efficacy still remains under investigation. In ulcerative colitis and Crohn's disease results with probiotics are less convincing or mixed ${ }^{[20-}$ $22,27,60]$. The results obtained with Bacillus subtilis 'PB6' in TNBS-induced colitis in rats, a well established, reliable model of human IBD, are suggestive of possible therapeutic effectiveness of PB6. 


\section{REFERENCES}

1. Lilly, D.M. and R.H. Sillwell, 1965. Probiotics: growth-promoting factors produced by microorganisms. Science, 147: 747-748.

2. Bergonzelli, G.E., S. Blum, H. Brüssow and I. Corthésy-Theulaz, 2005. Probiotics as a treatment strategy for gastrointestinal diseases? Digestion, 72: 57-68.

3. Hall, I.C. and E. O'Tool, 1935. Intestinal flora in newborn infants with a description of a new pathogenic anaerobe, Bacillus difficilis. Am. J. Dis. Child., 49: 390-402.

4. Kelly, C.P. and J.T. LaMont, 1998. Clostridium difficile infection. Annu. Rev., 49: 375-390.

5. Kelly, C.P., K. Pothoulakis and J.T. LaMont, 1994. Clostridium difficile colitis. N. Engl. J. Med., 330: 257-262.

6. Bartlett, J.G., 1992. Antibiotic associated diarrhea. Clin. Infect. Dis., 15: 573-581.

7. Fekety, R., 1995. Antibiotic associated colitis. In: Mandell, Douglas and Bennett's Principles of Infectious Diseases (Eds. G.L. Mandell, J.E. Bennett and E. Dolin) pp 978-987, 4th ed. Churchill Livingstone, New York.

8. Gerding, D.N., 1989. Disease associated with Clostridium difficile infection. Ann. Intern. Med., 110: 255-257.

9. Lyerly, D.M., H.C. Krivan and T.D. Wilkins, 1988. Clostridium difficile its disease and toxins. Clin. Microbiol. Rev., 1: 1-18.

10. McFarland, L.V. and W.E. Stamm, 1986. Review of Clostridium difficile-associated diseases. Am. J. Infect. Control, 14: 99-109.

11. Cetinkaya, Y., P. Falk and C.G. Mayhall, 2000. Vancomycin-resistant Enterococci. Clin. Microbiol. Rev., 13: 686-707.

12. Fekety, R., 1997. Guidelines for the diagnosis and management of Clostridium difficile-associated diarrhea and colitis. Am.J.Gastroenterol.,92: 739-750.

13. Wong, S.S., P.C. Woo, W.K. Luk and K.Y. Yuen, 1999. Susceptibility testing of Clostridium difficile against metronidazole and vancomycin by disk diffusion and E-test. Diagn. Micr. Infec. Dis., 34: 1-6.

14. Heerklotz, H. and J. Seelig, 2001. Detergent-like action of the antibiotic peptide surfactin on lipid membranes. Biophys. J., 81: 1547-1554.

15. Peypoux, F., J.M. Bonmatin and J. Wallach, 1999. Recent trends in the biochemistry of surfactin. Appl. Microbiol. Biotechnol., 51: 553-563.

16. Vollenbroich, D., G. Pauli, M. Ozel and J. Vater, 1997. Antimycoplasma properties and application in cell culture of surfactin, a lipopeptide antibiotic from Bacillus subtilis. Appl. Environ. Microbiol., 63: 44-49.

17. Vollenbroich, D., M. Ozel, J. Vater, R.M. Kamp and G. Pauli, 1997. Mechanism of inactivation of enveloped viruses by the biosurfactant surfactin from Bacillus subtilis. Biologicals, 25: 289-297.

18. Kameda, Y., S. Oira, K. Matsui, S. Kanatomo and T. Hase, 1974. Antitumor activity of Bacillus natto. V. Isolation and characterization of surfactin in the culture medium of Bacillus natto KMD 2311. Chem. Pharm. Bull., 22: 938-944.
19. Kim, K., S.Y. Jung, D.K. Lee, J.-K. Jung, J.K. Park, D.K. Kim and C.-H. Lee, 1998. Suppression of inflammatory responses by surfactin, a selective inhibitor of platelet cytosolic phospholipase A2. Biochem. Pharmacol., 55: 975-985.

20. Kruis, W., P. Fric, J. Pokrotnieks, M. Lukas, B. Fixa, M. Kascak, A. Kamm, J. Weismueller, C. Beglinger, M. Stolte, C. Wolff and J. Schulze, 2004. Maintaining remission of ulcerative colitis with the probiotic Escherichia coli Nissle 1917 is as effective as with standard mesalamine. Gut, 53: 1617-1623.

21. Bibiloni, R., R.N. Fedorak, G.W. Tannock, K.L. Madsen, P. Gionchetti, M. Campieri, C. De Simone and R.B. Sartor, 2005. VSL\#3 Probiotic-mixture induces remission in patients with active ulcerative colitis. Am. J. Gastroenterol., 100: 1539-1546.

22. Schultz, M., A. Timmer, H.H. Herfarth, R.B. Sartor, J.A. Vanderhoof and H.C. Rath, 2004. Lactobacillus $\mathrm{GG}$ in inducing and maintaining remission of Crohn's disease. BMC Gastroenterology, 4: 5.

23. Mimura, T., F. Rizello, U. Helwig, G. Poggioli, S. Schreiber, I.C. Talbot, R.J. Nicholls, P. Gionchetti, M. Campieri and M.A. Kamm, 2004. Once daily high dose probiotic therapy (VSL\#3) for maintaining remission in recurrent or refractory pouchitis. Gut, 53: 108-114.

24. Pena, J.A., A.B. Rogers, Z. Ge, V. Ng, S.Y. Li, J.G. Fox and J. Versalovic, 2005. Probiotic Lactobacillus spp. diminish Helicobacter hepaticus-induced inflammatory bowel disease in interleukine-10 deficient mice. Infect. Immun., 73: 912-920.

25. Farrell, R.J. and J.T. Lamont, 2003. Microbial factors in inflammatory bowel disease. Gastroenterol. Clin. North. Am., 31: 41-62.

26. Sartor, R.B., 1997. Enteric microflora in IBD: pathogens or commensals? Inflamm. Bowel Dis., 3: 230-235.

27. Sartor, R.B., 2004. Therapeutic manipulation of the enteric microflora in inflammatory bowel diseases: antibiotics, probiotics and prebiotics. Gastroenterology, 126: 1620-1633.

28. Tamboli, C.P., C. Neut, P. Desreumaux and J.F. Colombel, 2004. Dysbiosys in inflammatory bowel disease. Gut, 53: 1-4.

29. Papadakis, K.A. and S.R. Targan, 2000. Role of cytokines in the pathogenesis of inflammatory bowel disease. Annu. Rev. Med., 51: 289-298.

30. Bartlett, J.G., A.B. Onderdonk and R.L. Cisneros, 1977. Clindamycin-associated colitis in hamsters: protection with vancomycin. Gastroenterology, 73: 772-776.

31. Neurath, M., I. Fuss and W. Strober, 2000. TNBScolitis. Int. Rev. Immunol., 19: 51-62.

32. Duc, L.H., H.A. Hong, T.M. Barbosa, A.O Henriques and S.M. Cutting, 2004. Characterization of Bacillus probiotics available for human use. Appl. Environ. Microbiol., 70: 2161-2171. 
33. Kramer, C.Y., 1956. Extension of multiple range tests to group means with unequal numbers of replications. Biometrics, 12: 307-310.

34. Anton, P.M., M. O'Brien, E. Kokkotou, B. Eisenstein, A. Michaelis, D. Rothstein, S. Paraschos, C.P. Kelly and C. Pothoulakis, 2004. Rifalazil treats and prevents relapse of Clostridium difficile-associated diarrhea in hamsters. Antimicrob. Agents Chemother. 48: 3975-3979.

35. Browne, R.A., R. Fekety, J. Silva, D.I. Boyd, C.O. Work and G.D. Abrams, 1977. The protective effect of vancomycin on clindamycin-induced colitis in hamsters. Johns Hopkins Med. J., 141: 183-192.

36. McVay, C.S. and R.D. Rolfe, 2000. In vitro and in vivo activities of nitazoxanide against Clostridium difficile. Antimicrob. Agents Chemother., 44: 2254-2258.

37. Swanson, R.N., D.J. Hardy, N.L. Shipkowitz, C.W. Hanson, N.C. Ramer, P.B. Fernandes and J.J. Clement, 1991. In vitro and in vivo evaluation of tiacumicins $\mathrm{B}$ and $\mathrm{C}$ against Clostridium difficile. Antimicrob. Agents Chemother., 35: 1108-1111.

38. Elmer, G.W., 2001. Probiotics: "living drugs". Am. J. Health-Syst. Ph., 58: 1101-1109.

39. Sullivan, A. and C.E. Nord, 2002. Probiotics in human infections. J. Antimicrob. Chemoth., 50: 625-627.

40. Dendukuri, N., V. Costa, M. McGregor and J.M. Brophy, 2005. Probiotic therapy for the prevention and treatment of Clostridium difficile-associated diarrhea: a systematic review. Can. Med. Assoc. J., 173: 67-170.

41. Sambol, S.P., M.M. Merrigan, J.K. Tang, S. Johnson and D.N. Gerding, 2002. Colonization for the prevention of Clostridium difficile in hamsters. J. Infect. Dis., 186: 1781-1789.

42. Toothaker, R.D. and G.W. Elmer, 1984. Prevention of clindamycin-induced mortality in hamsters by Saccharomyces boulardii. Antimicrob. Agents Chemother., 26: 552-556.

43. Fabia, R., A. Ar'rajab, M.L. Johansson, R. Willen, R. Andersson, G. Molin and S. Bengmark, 1993. The effect of exogeneous administration of Lactobacillus reuteri R2LC and oat fiber on aceticacid-induced colitis in the rat. Scand. J. Gastroenterol., 28: 155-162.

44. Mao, Y., S. Nobaek, B. Kasravi, D. Adawi, U. Stenram, G. Molin and B. Jeppsson, 1996. The effects of Lactobacillus strains and oat fiber on methotrexate-induced enterocolitis in rats. Gastroenterology, 111: 334-344.

45. Madsen, K.L., 2001. Inflammatory bowel disease: lessons from the IL-10 gene-deficient mouse. Clin. Invest. Med., 24: 250-257.

46. Madsen, K.L., J.S. Doyle, L.D. Jewell, M.M. Taverini and R.N. Fedorak, 1999. Lactobacillus species prevents colitis in interleukin-10 genedeficient mice. Gastroenterology, 116: 1107-1114.

47. Kennedy, R.J., M. Hoper, K. Deodhar, S.J. Kirk and K.R. Gardiner, 2000. Probiotic therapy fails to improve gut permeability in a hapten model of colitis. Scand. J. Gastroenterol., 35: 1266-1271.

48. O’Mahony, L., M. Feeney, S. O'Halloran, L. Murphy, B. Kiely, K. Fitzgibbon, G. Lee, G. O'Sullivan, F. Shanahan F and J.K. Collins, 2001. Probiotic impact on microbial flora, inflammation and tumour development in IL-10 knock-out mice. Aliment. Pharmacol. Ther., 15: 1219-1225.
49. Schultz, M., C. Veltkamp, L.A. Dieleman, W.B. Grenther, P.B. Wyrick, S.L. Tonkonogy and R.B. Sartor, 2002. Lactobacillus plantarum 299v in the treatment and prevention of spontaneous colitis in interleukin-10 deficient mice. Inflamm. Bowel Dis., 8: 71-80.

50. Shibolet, O., F. Karmeli, R. Eliakim, E. Swennen, P. Brigidi, P. Gionchetti, M. Campieri, S. Morgenstern and D. Rachmilewitz, 2002. Variable response to probiotics in two models of experimental colitis in rats. Inflamm. Bowel Dis., 8: 399-406.

51. Woodruff, T.M., T.V. Arumugam, I.A. Shiels, R.C. Reid, P. Fairlie and S.M. Taylor, 2003. A potent human $\mathrm{C} 5 \mathrm{a}$ receptor antagonist protects against disease pathology in a rat model of inflammatory bowel disease. J. Immunol., 171: 5514-5520.

52. Hoa, T.T., L.H. Duc, R. Isticato, L. Baccigallupi, E. Ricca, P.H. Van and S.M. Cutting, 2001. Fate and dissemination of Bacillus subtilis spores in a murine model. Appl. Environ. Microbiol., 67: 3819-3823.

53. Van den Bosch, H., 1980. Intracellular phospholipases A. Biochim. Biophys. Acta, 604: 191-246.

54. Wallace, J.L. and L. Ma, 2001. Inflammatory mediators in gastrointestinal defense and injury. Exp. Biol. Med., 226: 1003-1015.

55. Waterman, W.H., T.F. Molski, C.K. Huang, J.L. Adams and R.L. Sha'afi,1996. Tumour necrosis factor-alpha-induced phosphorylation and activation of cytosolic phospholipase A2 are abrogated by an inhibitor of the p38 mitogenactivated protein kinase cascade in human neutrophils. Biochem. J., 319: 17-20.

56. Fabia, R., A. Ar'rajab, R. Willen, R. Andersson and S. Bengmark, 1993. Effect of putative phospholipase A2 inhibitors on acetic acid-induced acute colitis in the rat. Br. J. Surg., 80: 1199-1204.

57. Krimsky, M., M. Ligumsky, L. Aptekar, O. Schwob, G. Goshen, A. Gruzman, S. Sasson and S. Yedgar, 2003. Amelioration of TNBS-induced colon inflammation in rats by phospholipase $\mathrm{A}_{2}$ inhibitor. Am. J. Physiol. Gastrointest. Liver Physiol., 52: G586-G592.

58. Rioux, K.P. and R.N. Fedorak, 2006. Probiotics in the treatment of inflammatory bowel disease. J. Clin. Gastroenterol., 40: 260-263.

59. Ulisse, S., P. Gionchetti, S. D'Alo, F.P. Russo, I. Pesce, G. Ricci, F. Rizello, U. Helwig, M. Grazia Cifone, M. Campieri and C. De Simone, 2001. Expression of cytokines, inducible nitric oxide synthase and matrix metalloproteinases in pouchitis Effects of probiotic treatment. Am. J. Gastroenterol., 96: 2691-2699.

60. Prantera, C., M.L. Scribano, G. Falasco, A. Andreoli and C. Luzi, 2002. Ineffectiveness of probiotics in preventing recurrence after curative resection for Crohn's disease: a randomised controlled trial with Lactobacillus GG. Gut, 51: 405-409. 